\title{
BMJ Open Comparison of different mobile health applications for intervention in children and adolescent with overweight: a protocol for systematic review with meta-analysis and trial sequential analysis
}

\author{
Paolo Perego (1) , ${ }^{1}$ Rajeeb Rashid, ${ }^{2}$ Christian Gluud, ${ }^{3}$ Janus C Jakobsen, ${ }^{3,4}$ \\ Giuseppe Andreoni (D) , ${ }^{1}$ Inge Lissau (i) ${ }^{5}$
}

To cite: Perego P, Rashid R, Gluud C, et al. Comparison of different mobile health applications for intervention in children and adolescent with overweight: a protocol for systematic review with meta-analysis and trial sequential analysis. BMJ Open 2020;10:e032570. doi:10.1136/ bmjopen-2019-032570

- Prepublication history and additional material for this paper is available online. To view these files, please visit the journal online (http://dx.doi.org/10. 1136/bmjopen-2019-032570).

Received 25 June 2019 Revised 07 July 2020 Accepted 12 August 2020
Check for updates

(C) Author(s) (or their employer(s)) 2020. Re-use permitted under CC BY-NC. No commercial re-use. See rights and permissions. Published by BMJ.

For numbered affiliations see end of article.

Correspondence to

Dr Inge Lissau;

info@ingelissau.dk

\section{ABSTRACT}

Introduction Overweight in children is increasing worldwide. Innovative smartphone health applications (mHealth apps) have either sought to deliver single or multi-component interventions for the management of overweight in children. However, the clinical effects of these apps are poorly explored. The objective of the review will be to compare the benefits and harms of different categories of mHealth apps for intervention of overweight in children.

Methods and analysis We will include randomised clinical trials irrespective of publication type, year, status or language. Children and adolescents between 0 to 18 years will be referred to as children in the remaining part of the paper. Children with all degrees of overweight included obesity and morbidly obese in the remaining part of the paper will be referred to as overweight. We plan to classify different apps according to type of intervention, measurement device, coaching and reward system. The following databases will be used: Cochrane Library, Excerpta Medica database (Embase), PsycINFO, PubMed, IEEE Explore and Web of Science, CINAHL and LILACS. Primary outcomes will be body mass index zscore, quality of life and serious adverse event. Secondary outcomes will be body weight, self-efficacy, anxiety, depression and adverse event not considered serious. Study inclusion, data extraction and bias risk assessment will be conducted independently by at least two authors. We will assess the risk of bias through eight domains and control risks of random errors with Trial Sequential Analysis. The quality of the evidence will be assessed using Grading of Recommendations Assessment, Development and Evaluation Tool (GRADE).

Ethics and dissemination As the protocol is for a systematic reviews, we have not included any patient data and we do not require ethical approval. This review will be published in a peerreviewed journal.

PROSPERO registration number CRD42019120266.

\section{INTRODUCTION}

The prevalence of overweight are increasing worldwide among children irrespective of socio-economic status. ${ }^{1-3}$ By 2025 more than 260 million children aged 5 to 17 years may

\section{Strengths and limitations of this study}

- This review aims to be the first systematic review to compare the benefits and harms of different mobile health applications interventions in children with overweight following Cochrane guidelines.

- A comprehensive search strategy will be used with a large number of databases searched, and only randomised controlled trials in children with overweight will be included.

- The review will perform meta-analysis, Trial Sequential Analysis and use the Grading of Recommendations Assessment, Development and Evaluation Tool.

- We expect high heterogeneity across studies which may lead to challenges in performing a meta-analysis.

- It is anticipated that many papers will not provide sufficient details on all variables of interest and will lead to reliance on communication with corresponding authors for additional information.

be overweight, including 91 million obese according to data from Global Burden of Disease collaborative for 2000 and $2013 .{ }^{45}$ The International Task Force of Obesity produced age and gender specific cut-off for the definition of overweight and obesity in children. ${ }^{6}$ Throughout this paper we will use the term overweight for all children with overweight including all levels of obesity.

These trends of increasing overweight will have long-term consequences on cardiovascular disease, insulin resistance, type 2 diabetes and cancer (endometrial, breast and colon), resulting in a significant burden on health services across the world. ${ }^{4}$ Recently, there has been an exponential growth in connected 


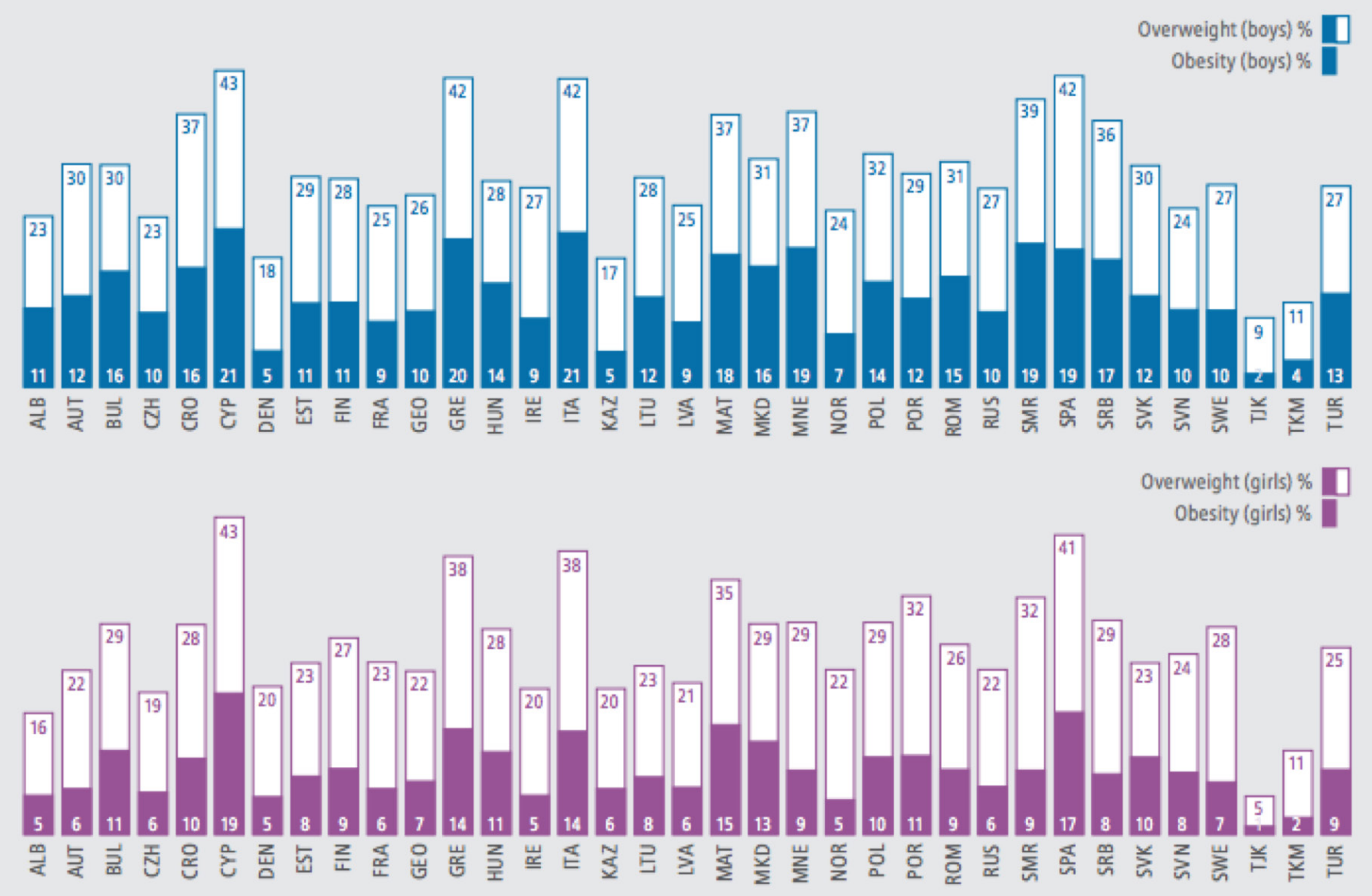

Figure 1 Overweight and obesity prevalence values based on WHO definition - COSI 2015 to $2017^{4}$.

devices such as smartphones and tablets, supporting the development of new services. A plethora of software applications (apps) running on these devices are appearing on the market (figure 1). Health apps thus represent a huge area which potentially exploits the new paradigm of mHealth-Mobile Health. This refers to medicine and public health services supported by mobile devices. mHealth apps are commonly used in disease surveillance, treatment support and for educating children about prevention. However, despite the potential opportunities of mHealth, the main issue of these applications is the ability to engage the users to keep them motivated using the app, an aspect that is even more difficult if the users are young.

Children are 'millennials' and 'natively digital' hence mobile technologies are potentially relevant and accessible tools for them, even in their health management. In this paper, we combined these two elements to analyse the mutual inter-relationships between the use of mobile systems to counteract overweight in children. Recent Cochrane reviews highlighted the benefits of multi-component interventions over single approach programmes on treating children with overweight. The authors, however, noted the paucity of good quality trials on multi-component interventions. ${ }^{8-10}$

\section{mHealth apps}

Smartphones increase the possibility to interact with people in a more personalised and tailored manner. They enable the building of platforms for adaptive interventions with visually appealing and engaging multimedia modalities which can be adjusted by the user based on their preferences. ${ }^{11}$ mHealth apps have the ability to support children to achieve and maintain a healthy and sustainable lifestyle by supporting and strengthening their self-regulatory capacities. ${ }^{12} 13$ They offer potential advantages over traditional face-to-face methods for delivering health-related interventions. ${ }^{14-17}$ These include cost-effective dissemination, real-time data collection and feedback, lowered participant burden and flexible programme tailoring. ${ }^{11}$

No reviews to date, to our best knowledge, have specifically compared the efficacy of different categories of apps for interventions in children with overweight. In addition, while previous reviews have commented on the significant risk of bias in many studies, there has not been a consistency in including control of bias, the play of chance and assessing the quality with GRADE (Grading of Recommendations Assessment, Development and Evaluation) assessment in these reviews. ${ }^{18-27}$

Nutrition and diet apps represent a popular area of mHealth, offering the possibility of delivering behavioural change interventions for healthy eating and weight management in a scalable and cost-effective way. Use of commercial apps for paediatric weight management often fail to retain users because of a lack of theoretical 
background and evidence-based content. However, mHealth apps that are more evidence-based are often found less engaging and popular among consumers. ${ }^{28}$ Approaching the apps development process from a multidisciplinary, expert and user-centred design perspective is more likely to help overcome these limitations. They may also provide easier adoption and integration of nutritional education apps within primary and secondary care interventions. ${ }^{29} 30$

Such a process has been transitioned into health game apps where long-term use is dependent on providing easy and continual gaming access on both smartphones and tablets; offer games that can be personalised and are adaptable based on the child's interests; and maintain novelty and interest in the treatment over time. ${ }^{31}$ This framework not only provides a benefit to the children involved, but also provides user data to the coaches, clinicians and health researchers involved in the child's treatment regime. ${ }^{32}$ In addition, while many apps tended to focus either on nutrition or on physical activity, very few apps managed to adhere to or deliver a comprehensive overweight intervention for children due to a failure to support a spectrum of important target behaviours. ${ }^{33}$

The role of health researchers is therefore to evaluate the evidence base, efficacy and quality of different apps to ascertain whether these apps may have a part to play in the management of childhood overweight. In addition, assessment of the effect of the interventions have not been evaluated taking risks of bias, risks of random errors, type of control interventions, as well as the quality of evidence into account. ${ }^{34}$

\section{Objective}

The objective of the review will be to compare the benefits and harms of different categories of mHealth apps in interventions for overweight in children.

\section{METHODS AND ANALYSIS}

This work consists in a protocol for systematic review with meta-analysis and Trial Sequential Analysis with the aim to compare different typology of mobile health applications for intervention in children and adolescent with overweight. The paper is a continuation of the previous work ${ }^{35}$ by the same authors in which the effectiveness of the use of applications in the prevention of obesity was studied. The methods section overlaps in part with our previous publication and with other Cochrane protocols and reviews, especially those following Cochrane methodology and using Trial Sequential Analysis which have common authors (C Gluud and J C Jakobsen).

\section{Criteria for considering studies for this review}

\section{Types of studies}

Randomised clinical trials irrespective of language, publication status, publication type or publication year up to July 2020 will be included in the review. Eligible studies which are not published in English will be translated using
Google translate. Authors will be contacted to check if an English translation is available or to clarify any queries.

\section{Types of participants}

All children who are overweight (including all levels of obesity) up to 18 years of age (as defined earlier). Children with associated comorbidities, either physical or psychological secondary to overweight and obesity will be included. Children with causes of overweight due to medication such as steroids, or genetic disorders which are associated with overweight will be excluded.

\section{Types of intervention}

Any type of mHealth intervention using apps. There is no restriction as to how the app delivers the intervention or intervention duration or the type of electronic platform (smartphones, tablets and so on).

\section{Types of outcomes}

We will assess at baseline and then all outcomes at two further time points:

- End of intervention: as defined by trialist (primary time point of interest).

- Maximum follow-up.

\section{Primary outcomes}

- BMI z-score.

- Quality of life: as measured by any scale that has been validated for use in the target population. ${ }^{36}$

- Proportion of participants with at least one serious adverse event. ${ }^{34}$

\section{Secondary outcomes}

- Body weight measured in $\mathrm{kg}$.

- Self-efficacy: as measured by a scale validated for use in children.

- Anxiety.

- Depression.

- Proportion of participants with at least one adverse event not considered serious.

\section{Exploratory outcomes}

- Body fat measured by bioimpedance or dual energy x-ray absorptiometry(DEXA), there having been good correlation between total body fat $\%$ by bioimpedance or DEXA $(\mathrm{r}=0.87, \mathrm{p}<0.001) .{ }^{3738}$

- Muscle mass (kg) via bioimpedance or DEXA. ${ }^{37} 38$

- Individual serious and non-serious adverse events.

\section{Primary classification of mHealth apps}

mHealth app interventions in overweight children cover a variety of typologies and related strategies. We have subsequently provided a systematic categorisation of these approaches for the systematic review. While appreciating that there will be some overlap in the app characteristics, the categorisation aims to identify the primary purpose or key component of the app in the intervention.

mHealth apps for overweight interventions usually target three different strategies-dietary change, increase 
in physical activity and behavioural change. ${ }^{39}$ Apps are mainly targeted to monitor or motivate small improvements such as steps per day, duration and intensity of physical activity or counting calories for nutrition. Indeed, behaviour or lifestyle (which also includes nutrition and physical activity) integrates data and suggests activities to the users in order to motivate them and try to change their behaviour.

Based on these three strategies, the apps can be then divided into different categories according to the main characteristic which is listed below as main bullet points:

- Presence of devices

- Standalone mobile applications without connected devices for data gathering.

- Mobile applications with devices (wearable devices, smart scales and so on).

- Coach

- Mobile applications with a real human coach who interacts with the users (phone calls or messages).

- Mobile applications with a virtual coach which provides suggestions to the users by means of gathered data.

- Mobile application without a coach: this app only gets data and shows them to the users.

- Intervention (only if app includes a coach)

- Mobile application with a standard reminder/suggestion, like the standard calendar notification.

- Mobile application with an intelligent reminder/ suggestion based on acquired data and habits.

- Mobile application without a direct intervention.

Reward

- Mobile application with an intangible reward (virtual coins for in app purchase, emoji).

- Mobile application with a tangible reward (money or discount coupons). ${ }^{40}$

- Mobile application without a reward.

Further app categories can be described based on the connection of the application and how users can download it.

- Cloud and social connectivity.

- Mobile application connected with the cloud to store and process data.

- Standalone application without a connection.

Online supplemental table 1 shows this app classification with some examples and scientific papers related to them.

\section{Search methods for identification of studies}

\section{Electronic searches}

We will search the following databases:

- Cochrane Library.

- MEDLINE.

- Excerpta Medica database (Embase).

- PsycINFO.

- IEEE Explore.

- Web of Science (SCI-EXPANDED, SSCI, A\&HCI, CPCI-S, CPCI-SSH, ESCI, CCR-EXPANDED, IC).

- CINAHL.
- LILACS.

Searching other resources

- ClinicalTrials.gov (http://www.clinicaltrials.gov/)

- Google Scholar (https://scholar.google.com/)

- European Medicine Agency (http:// www.ema. europa.eu/ema/)

- United States Food and Drug Administration (www. fda.gov)

- Medicines and Healthcare Products Regulatory Agency (https://www.gov.uk/government/organisations/medicines-and-healthcare-products-regulatoryagency)

- The WHO (www.who.int/)

- Global Obesity Forum (previously International Association for the study of Obesity) (www.iaso.org)

- European Association for the study of Obesity (EASO) (https://easo.org/)

- ICTRP Search Portal used in the search strategy

Keywords used in the search strategy

- Obesity

- Overweight

- Smartphone apps

- Health apps

- mHealth app

- Body mass index

- Weight gain

- Weight loss

- Hyperphagia

- Randomised controlled trial

Preliminary search strategy for MEDLINE is enclosed as online supplemental additional file 2 .

\section{Data collection and analysis}

Selection of studies

The review will follow the recommendations in the Cochrane Handbook for Systematic Reviews of Interventions and according to Keus et al and Jakobsen et al. ${ }^{4142}$ The analyses will be performed using Review Manager ${ }^{43}$ and Trial Sequential Analysis programme. ${ }^{44}$

Two authors (RR and PP) will independently screen titles and abstracts. They will retrieve all relevant fulltext study/publication after which two authors will independently screen the full text in order to identify and record reasons for exclusion of the ineligible studies. We will resolve any disagreement through discussion. Trial selection will be displayed in an adapted flow diagram as per the Preferred Reporting Items for Systematic Reviews and Meta-Analyses (PRISMA) statement. Online supplemental additional file 3 reports the PRISMA checklist.

\section{Data extraction and management}

Data extraction will be performed independently by at least two authors, who will both compare the extracted data. Disagreements will be resolved by a third author. We will assess duplicate publications and companion papers of a trial together to evaluate all available data simultaneously (maximise data extraction and correct 
bias assessment). Trial authors will be contacted by email to request any additional data which may not have been reported sufficiently or at all in the publication. Review Manager software will be used to extract data.

\section{Assessment of risk of bias in included studies}

The risk of bias of every included trial will be evaluated independently by at least two authors. In case of any disagreement, discrepancies will be discussed with a third author and resolved by consensus. The risk of bias will be assessed using the Cochrane's 'Risk of bias' assessment tool $^{4546}$ and the Cochrane Effective Practice and Organisation of Care Group's guidance. ${ }^{46}$ We will evaluate the methodology in respect of:

- Random sequence generation.

- Allocation concealment.

- Blinding of participants and treatment providers.

- Blinding of outcome assessment.

- Incomplete outcome data.

- Selective outcome reporting.

- For profit bias.

- Overall risk of bias.

Classification of the trials will follow criteria defined in online supplemental file 4.

\section{Meta-analysis}

Both end-scores and change-from-baseline scores will be used to analyse continuous outcomes. If both end-scores and change-from-baseline scores are reported then only end-scores will be used. If only change-from-baseline scores are reported, these results together with end-scores will be analysed in the same meta-analyses. ${ }^{45}$ Exploratory outcomes will be analysed using change-from-baseline scores.

Data will be meta-analysed by RevMan 5 statistical software. We will use Stata statistical software (Stata 2015) in case of zero event trials, where RevMan 5 zero event handling is insufficient. ${ }^{478}$ We will report effect estimate using mean difference with $95 \%$ CIs.

Intervention effects will be assessed by both randomeffects model meta-analyses and fixed-effect model metaanalyses, ${ }^{27449}$ using the more conservative point estimate of the two. Three primary outcomes will be examined with $\mathrm{p} \leq 0.025$ being statistically significant. An eight-step procedure will be used to assess if the thresholds for significance are crossed. Five secondary outcomes will be examined with $\mathrm{p} \leq 0.017$ being statistically significant. ${ }^{38}$ The results of the exploratory outcomes will be considered hypothesis generating only.

Analysis of all included studies will be compared with a sensitivity analysis of studies at low risk of bias. If the results are similar, primary conclusions will be based at the time point closest to 12 months on the overall analysis. If the results differ, primary conclusions will be based on studies with a low risk of bias.

A table describing the types of serious adverse events in each trial will be provided.

\section{Trial sequential analysis}

Traditional meta-analysis runs the risk of random errors due to sparse data and repetitive testing of accumulating data when updating reviews. Trial Sequential Analysis will thus be used to analyse the outcomes in order to calculate the required information size and control the risks of type I errors and type II errors. ${ }^{26} 2748$

For continuous outcomes, Trial Sequential Analysis will use the observed SD, a mean difference of the observed $\mathrm{SD} / 2$, an alpha of $2.5 \%$ for the three primary outcomes, an alpha of $1.67 \%$ for the five secondary outcomes and a beta of $10 \%$, with adjustment for observed diversity. ${ }^{50}$ Mean differences (MDs) and the standardised mean difference will be expressed with $95 \%$ CIs for continuous outcomes, as well as the Trial Sequential Analysis adjusted CIs for MDs. We intend to use the proportion in the control group and the diversity estimated in the meta-analysis to provide reliable results.

For dichotomous outcomes, Trial Sequential Analysis will use the proportion of participants with an outcome in the control group, a relative risk reduction of $20 \%$, an alpha of $2.5 \%$ for primary outcomes, an alpha of $1.67 \%$ for secondary outcomes and a beta of $10 \%$, with adjustment for observed diversity. We will calculate risk ratios with $95 \%$ CI for dichotomous outcomes, as well as Trial Sequential Analysis adjusted CIs.

\section{Subgroup analyses}

Subgroup analysis when analysing the primary outcomes will be performed as follows:

- Trials at high risk of bias trials compared to trials at low risk of bias trials.

- Trials stratified according to experimental interventions.

- Trials stratified according to weight status: overweight, obese or morbidly obese at the entry into the trial. ${ }^{6}$

- Trial stratified according to the control interventions.

- Complexity: trials with participants with no comorbidities compared to trials with participants pre-existing comorbidities.

- Trials in which the experimental intervention was evaluated by either the parents or the child after the treatment sessions had been delivered compared to trials in which the experimental intervention was not evaluated by either the parents or the child after the treatment sessions had been delivered.

We will use the formal test for subgroup interactions in Review Manager. ${ }^{43}$

\section{Sensitivity analysis}

To assess the potential impact of the missing data for dichotomous outcomes, we will perform the following sensitivity analyses:

- 'Best-worst-case' scenario: we will assume that all participants lost to follow-up in the experimental group had no serious adverse events, including not developing any psychiatric disease such as an eating disorder. 
- 'Worst-best-case' scenario: all dropouts/participants lost from the experimental group, but none from the control group experienced the outcome, including all randomised participants in the denominator.

Statistical heterogeneity will be assessed by visual inspection of the forest plots and $\mathrm{I}^{2}$ statistic values. ${ }^{41}$ Underlying reasons behind statistical heterogeneity in meta-analyses will be investigated by assessing trial characteristics.

\section{Summary of findings table}

A summary of findings table using each of the prespecified primary and secondary outcomes will be reported using the GRADE considerations for studies which contribute data to the meta-analyses for the prespecified outcomes. ${ }^{34} 41$ 52-65 Methods and recommendations described in Chapter 8 (Section 8.5) and Chapter 12 of the Cochrane Handbook for Systematic Reviews of Interventions $^{66}$ will be followed using GRADEpro software.

\section{DISCUSSION}

The objective of the review will be to compare the benefits and harms of different categories of mHealth apps for intervention of overweight in children. Currently, there are no systematic reviews which specifically compare the effects of different typology of mHealth apps to interventions in children with overweight. Previous systematic reviews in children have considered the efficacy of mobile health technologies more broadly in the role of weight management, ${ }^{65}$ but none have provided comprehensive coverage of the benefits and harms of mHealth apps nor an in-depth study of the different types of apps. Hence, this evidence will hopefully help children and adolescents, their parents and health professionals to make informed treatment decisions. This review will also highlight any gaps in the evidence base of such interventions and in app structure which will help to shape the development and optimisation of future potential interventions and apps.

\section{Author affiliations}

${ }^{1}$ Design Department, Politecnico di Milano, Milano, Ml, Italy

${ }^{2}$ Department of Child Life and Health, The University of Edinburgh, Edinburgh, UK

${ }^{3}$ The Copenhagen Trial Unit, Centre for Clinical Intervention Research,

Rigshospitalet, Copenhagen University Hospital, Copenhagen, Denmark

${ }^{4}$ Department of Cardiology, Holbaek Sygehus, Holbaek, Sjaelland, Denmark

${ }^{5}$ Clinical Research Centre, University Hospital Copenhagen, Copenhagen, Hvidovre, Denmark

\section{Twitter Paolo Perego @pperego}

Acknowledgements We would like to thank Sarah Louise Klingenberg, Information Specialist at the Copenhagen Trial Unit, Centre for Clinical Intervention Research, for her guidance and assistance on developing search methods keywords for search strategies.

Contributors IL, RR, PP, GA, CG and JCJ wrote the first draft of the protocol. PP, $\mathrm{RR}, \mathrm{IL}, \mathrm{GA}, \mathrm{JCJ}$ and CG have revised the protocol. All authors read and approved the manuscript. IL is the guarantor for the review.

Funding The authors have not declared a specific grant for this research from any funding agency in the public, commercial or not-for-profit sectors.

Competing interests None declared.
Patient consent for publication Not required.

Provenance and peer review Not commissioned; externally peer reviewed.

Supplemental material This content has been supplied by the author(s). It has not been vetted by BMJ Publishing Group Limited (BMJ) and may not have been peer-reviewed. Any opinions or recommendations discussed are solely those of the author(s) and are not endorsed by BMJ. BMJ disclaims all liability and responsibility arising from any reliance placed on the content. Where the content includes any translated material, BMJ does not warrant the accuracy and reliability of the translations (including but not limited to local regulations, clinical guidelines, terminology, drug names and drug dosages), and is not responsible for any error and/or omissions arising from translation and adaptation or otherwise.

Open access This is an open access article distributed in accordance with the Creative Commons Attribution Non Commercial (CC BY-NC 4.0) license, which permits others to distribute, remix, adapt, build upon this work non-commercially, and license their derivative works on different terms, provided the original work is properly cited, appropriate credit is given, any changes made indicated, and the use is non-commercial. See: http://creativecommons.org/licenses/by-nc/4.0/.

\section{ORCID iDs}

Paolo Perego http://orcid.org/0000-0003-4960-8888

Giuseppe Andreoni http://orcid.org/0000-0002-5537-4128

Inge Lissau http://orcid.org/0000-0002-2225-9975

\section{REFERENCES}

1 Wang Y, Lobstein T. Worldwide trends in childhood overweight and obesity. Int J Pediatr Obes 2006;1:11-25.

2 Lobstein T. The prevention of obesity in children. Pediatr Endocrinol Rev 2004;1 Suppl 3:471-5.

3 de Onis M, Blössner M, Borghi E. Global prevalence and trends of overweight and obesity among preschool children. Am J Clin Nutr 2010;92:1257-64.

4 Organisation WH. COSI: childhood obesity surveillance initiative. Copenhagen: WHO, 2018.

5 Organisation WH. Obesity and overweight. Copenhagen, 2016. Available: http://www.who.int/mediacentre/factsheets/fs311/en/

6 Cole TJ, Bellizzi MC, Flegal KM, et al. Establishing a standard definition for child overweight and obesity worldwide: international survey. BMJ 2000;320:1240-3.

7 Short CE, DeSmet A, Woods C, et al. Measuring engagement in eHealth and mHealth behavior change interventions: viewpoint of methodologies. J Med Internet Res 2018;20:e292.

8 Colquitt JL, Loveman E, O'Malley C, et al. Diet, physical activity, and behavioural interventions for the treatment of overweight or obesity in preschool children up to the age of 6 years. Cochrane Database Syst Rev 2016;3:Cd012105.

9 Al-Khudairy L, Loveman E, Colquitt JL, et al. Diet, physical activity and behavioural interventions for the treatment of overweight or obese adolescents aged 12 to 17 years. Cochrane Database Syst Rev 2017;6:CD012691.

10 Mead E, Brown T, Rees K, et al. Diet, physical activity and behavioural interventions for the treatment of overweight or obese children from the age of 6 to 11 years. Cochrane Database Syst Rev 2017:6:Cd012651.

11 Tate EB, Spruijt-Metz D, O'Reilly G, et al. mHealth approaches to child obesity prevention: successes, unique challenges, and next directions. Trans/ Behav Med 2013;3:406-15.

12 Anderson J, Kamphorst B. Ethics of e-coaching: implications of employing pervasive computing to promote healthy and sustainable lifestyles, 2014: 351-6.

13 Baroni I, Nalin M, Coti Zelati M. Designing motivational robot: how robots might motivate children to eat fruits and vegetables, 2014

14 Andreoni G, Perego P, Frumento E. $m \_$Health Current and Future Applications. (EAl/Springer Innovations in Communication and Computing. Cham: Springer International Publishing, 2019. http:// link.springer.com/10.1007/978-3-030-02182-5

15 Bardus M, Smith JR, Samaha L, et al. Mobile phone and web 2.0 technologies for weight management: a systematic scoping review. $J$ Med Internet Res 2015;17:e259.

16 Flores Mateo G, Granado-Font E, Ferré-Grau C, et al. Mobile phone Apps to promote weight loss and increase physical activity: a systematic review and meta-analysis. J Med Internet Res 2015;17:e253.

17 Badawy SM, Kuhns LM. Texting and mobile phone APP interventions for improving adherence to preventive behavior in adolescents: a systematic review. JMIR Mhealth Uhealth 2017;5:e50. 
18 Kjaergard LL, Villumsen J, Gluud C. Reported methodologic quality and discrepancies between large and small randomized trials in meta-analyses. Ann Intern Med 2001;135:982-9.

19 Schünemann HJ, Wiercioch W, Brozek J, et al. Grade evidence to decision (ETD) frameworks for adoption, adaptation, and de novo development of trustworthy recommendations: GRADEADOLOPMENT. J Clin Epidemiol 2017;81:101-10.

20 Savović J, Jones HE, Altman DG, et al. Influence of reported study design characteristics on intervention effect estimates from randomized, controlled trials. Ann Intern Med 2012;157:429.

21 Savović J, Jones H, Altman D, et al. Influence of reported study design characteristics on intervention effect estimates from randomised controlled trials: combined analysis of metaepidemiological studies. Health Technol Assess 2012;16:1-82.

22 Schulz KF, Chalmers I, Hayes RJ, et al. Empirical evidence of bias. dimensions of methodological quality associated with estimates of treatment effects in controlled trials. JAMA 1995;273:408-12.

23 Wood L, Egger M, Gluud LL, et al. Empirical evidence of bias in treatment effect estimates in controlled trials with different interventions and outcomes: meta-epidemiological study. BMJ 2008;336:601-5.

24 Lundh A, Sismondo S, Lexchin J, et al. Industry sponsorship and research outcome. Cochrane Database Syst Rev 2012;12:Mr000033.

25 Moher D, Pham B, Jones A, et al. Does quality of reports of randomised trials affect estimates of intervention efficacy reported in meta-analyses? Lancet 1998;352:609-13.

26 Wetterslev J, Jakobsen JC, Gluud C. Trial sequential analysis in systematic reviews with meta-analysis. BMC Med Res Methodol 2017;17:39.

27 Wetterslev J, Thorlund K, Brok J, et al. Estimating required information size by quantifying diversity in random-effects model meta-analyses. BMC Med Res Methodol 2009;9:86.

28 Boulos MNK, Brewer AC, Karimkhani C, et al. Mobile medical and health apps: state of the art, concerns, regulatory control and certification. Online J Public Health Inform 2014;5:229.

29 Gabrielli S, Dianti M, Maimone R, et al. Design of a mobile APP for nutrition education (TreC-LifeStyle) and formative evaluation with families of overweight children. JMIR Mhealth Uhealth 2017;5:e48.

$30 \mathrm{Kim}$ KK, Logan HC, Young E, et al. Youth-centered design and usage results of the iN touch mobile self-management program for overweight/obesity. Pers Ubiquitous Comput 2015;19:59-68.

31 Ronimus M, Kujala J, Tolvanen A, et al. Children's engagement during digital game-based learning of reading: The effects of time, rewards, and challenge. Comput Educ 2014;71:237-46.

32 Watters C, Author M, Abouzied A. Extending the use of games in health care. 5, 2006

33 Breton ER, Fuemmeler BF, Abroms LC. Weight loss-there is an APP for that! but does it adhere to evidence-informed practices? Trans/ Behav Med 2011;1:523-9.

34 Garattini S, Jakobsen JC, Wetterslev J, et al. Evidence-Based clinical practice: overview of threats to the validity of evidence and how to minimise them. Eur J Intern Med 2016;32:13-21.

35 Rashid R, Perego P, Condon L, et al. Health apps targeting children with overweight-a protocol for a systematic review with metaanalysis and trial sequential analysis of randomised clinical trials. Syst Rev 2020;9:28.

36 Ahuja B, Klassen AF, Satz R, et al. A review of patient-reported outcomes for children and adolescents with obesity. Qual Life Res 2014;23:759-70.

37 Bandura A. Social foundations of thought and action: a social cognitive theory. Englewood Cliffs: Prentice-Hall, 1986.

38 Murtagh J, Dixey R, Rudolf M. A qualitative investigation into the levers and barriers to weight loss in children: opinions of obese children. Arch Dis Child 2006;91:920-3.

39 Puigdomenech E, Martin A, Lang A, et al. Promoting healthy teenage behaviour across three European countries through the use of a novel smartphone technology platform, PEGASO fit for future: study protocol of a quasi-experimental, controlled, multi-centre trial. BMC Med Inform Decis Mak 2019;19:278.

40 Pedersen C, Holland A. Smart technology for health and well-being. $j$ fam consum sci 2018;110:55-6.
41 Jakobsen JC, Wetterslev J, Winkel P, et al. Thresholds for statistical and clinical significance in systematic reviews with meta-analytic methods. BMC Med Res Methodol 2014;14:120.

42 Keus F, Wetterslev J, Gluud C, et al. Evidence at a glance: error matrix approach for overviewing available evidence. BMC Med Res Methodol 2010;10:90.

43 The Cochrane Collaboration. Review manager. Copenhagen: Nordic Cochrane Centre, 2014.

44 Thorlund K, Wetterslev J, Brok J, et al. User manual for trial sequential analysis (TSA), 2011. Available: https://www.ctu.dk/tsa/

45 Davey J, Turner RM, Clarke MJ, et al. Characteristics of metaanalyses and their component studies in the Cochrane database of systematic reviews: a cross-sectional, descriptive analysis. BMC Med Res Methodol 2011;11:160.

46 Riley RD, Higgins JPT, Deeks JJ. Interpretation of random effects meta-analyses. BMJ 2011;342:d549.

47 Demets DL. Methods for combining randomized clinical trials: strengths and limitations. Stat Med 1987;6:341-8.

48 Wetterslev J, Thorlund K, Brok J, et al. Trial sequential analysis may establish when firm evidence is reached in cumulative meta-analysis. $J$ Clin Epidemiol 2008;61:64-75

49 Keus F, Wetterslev J, Gluud C, et al. Trial sequential analyses of meta-analyses of complications in laparoscopic vs. small-incision cholecystectomy: more randomized patients are needed. J Clin Epidemiol 2010;63:246-56.

50 European Commission. Proposal for a good clinical practice Directive. Bull Med Ethics 1998;No. 135:6-11.

51 StataCorp. StataCorp statistical software. release 14. College Station: StataCorp Lp, 2014. Available: http://www.stata.com

52 Guyatt GH, Oxman AD, Vist GE, et al. Grade: an emerging consensus on rating quality of evidence and strength of recommendations. $B M J$ 2008;336:924-6.

53 Schünemann HJ, Best D, Vist G, et al. Letters, numbers, symbols and words: how to communicate grades of evidence and recommendations. CMAJ 2003;169:677-80.

54 Guyatt GH, Oxman AD, Schünemann HJ, et al. Grade guidelines: a new series of articles in the Journal of clinical epidemiology. J Clin Epidemiol 2011;64:380-2.

55 Atkins D, Best D, Briss PA, et al. Grading quality of evidence and strength of recommendations. BMJ 2004;328:1490.

56 Guyatt G, Oxman AD, Akl EA, et al. Grade guidelines: 1. IntroductionGRADE evidence profiles and summary of findings tables. J Clin Epidemiol 2011;64:383-94.

57 Guyatt GH, Oxman AD, Kunz R, et al. Grade guidelines: 2. framing the question and deciding on important outcomes. J Clin Epidemiol 2011;64:395-400.

58 Guyatt GH, Oxman AD, Vist G, et al. GRADE guidelines: 4. Rating the quality of evidence--study limitations (risk of bias). J Clin Epidemiol 2011;64:407-15

59 Guyatt GH, Oxman AD, Montori V, et al. GRADE guidelines: 5. Rating the quality of evidence--publication bias. J Clin Epidemiol 2011;64:1277-82.

60 Guyatt GH, Oxman AD, Kunz R, et al. GRADE guidelines: 7. Rating the quality of evidence--inconsistency. J Clin Epidemiol 2011;64:1294-302.

61 Guyatt GH, Oxman AD, Kunz R, et al. GRADE guidelines: 8. Rating the quality of evidence--indirectness. J Clin Epidemiol 2011;64:1303-10.

62 Guyatt GH, Oxman AD, Sultan S, et al. Grade guidelines: 9. rating up the quality of evidence. J Clin Epidemiol 2011;64:1311-6.

63 Guyatt G, Oxman AD, Sultan S, et al. Grade guidelines: 11. making an overall rating of confidence in effect estimates for a single outcome and for all outcomes. J Clin Epidemiol 2013;66:151-7.

64 Guyatt GH, Oxman AD, Santesso N, et al. Grade guidelines: 12 preparing summary of findings tables-binary outcomes. J Clin Epidemiol 2013;66:158-72.

65 Darling KE, Sato AF, Review S. Systematic review and meta-analysis examining the effectiveness of mobile health technologies in using self-monitoring for pediatric weight management. Child Obes 2017;13:347-55

66 Chandler J, Cumpston M, Li T, et al. Cochrane Handbook for systematic reviews of interventions. John Wiley \& Sons, 2019. 